\title{
SOME CALKIN ALGEBRAS HAVE OUTER AUTOMORPHISMS
}

\author{
ILIJAS FARAH, PAUL MCKENNEY, AND ERNEST SCHIMMERLING
}

\begin{abstract}
We consider various quotients of the $\mathrm{C}^{*}$-algebra of bounded operators on a nonseparable Hilbert space, and prove in some cases that, assuming some restriction of the Generalized Continuum Hypothesis, there are many outer automorphisms.
\end{abstract}

\section{INTRODUCTION}

Let $\mathcal{H}$ be a Hilbert space. The Calkin algebra over $\mathcal{H}$ is the quotient $\mathcal{C}(\mathcal{H})=\mathcal{B}(\mathcal{H}) / \mathcal{K}$, where $\mathcal{B}(\mathcal{H})$ is the $\mathrm{C}^{*}$-algebra of bounded, linear operators on $\mathcal{H}$, and $\mathcal{K}$ is its ideal of compact operators. Assuming the Continuum Hypothesis, Phillips and Weaver constructed $2^{2^{\aleph_{0}}}$-many automorphisms of the Calkin algebra on the Hilbert space of dimension $\left.\aleph_{0}(4]\right)$. Since there are only $2^{\aleph_{0}}$-many automorphisms of $\mathcal{C}(\mathcal{H})$ which are inner (that is, implemented by conjugation by a unitary), this implies in particular that there are many more outer automorphisms than there are inner ones, in the presence of $\mathrm{CH}$.

The first author proved in [2] that it is relatively consistent with ZFC that all automorphisms of the Calkin algebra on a separable Hilbert space are inner. This establishes the existence of an outer automorphism as a question independent of ZFC. The assumption made there was Todorčević's Axiom (TA), a combinatorial principle also known as the Open Coloring Axiom. TA has a number of consequences in other areas of mathematics, and follows from the Proper Forcing Axiom (PFA), which is itself well-known for its influence on certain kinds of rigidity in mathematics (see [3]). The first author extended this result to prove that all automorphisms of the Calkin algebra over any Hilbert space, separable or not, are inner, assuming PFA ([1]).

The development of these results parallels those in the study of the automorphisms of the Boolean algebra $\mathcal{P}(\omega)$ /fin. Rudin ([5]) discovered early on that, assuming $\mathrm{CH}$, there are many automorphisms of $\mathcal{P}(\omega) /$ fin that are not trivial, i.e. induced by functions $e: \omega \rightarrow \omega$; Shelah ([6]) much later proved the consistency of the opposite result, that all automorphisms are trivial. Shelah and Steprans then showed that all automorphisms are trivial

This work was initiated at the Mittag-Leffler Institute during the authors' visit in September, 2009. The first author was partially supported by NSERC. 
assuming PFA ([7]), and then Veličković showed using PFA that all automorphisms of $\mathcal{P}(\kappa) /$ fin are trivial, for every infinite cardinal $\kappa$ (along with reducing the assumption to $\mathrm{TA}+\mathrm{MA}_{\aleph_{1}}$ in the original case $\left.\kappa=\omega\right)$.

One might ask for the consistency of outer automorphisms of $\mathcal{C}(\mathcal{H})$ when $\mathcal{H}$ is nonseparable, or nontrivial automorphisms of $\mathcal{P}(\kappa) /$ fin when $\kappa$ is uncountable. The latter result is easy, though for trivial reasons, since the automorphisms of $\mathcal{P}(\omega) /$ fin can all be extended to automorphisms of $\mathcal{P}(\kappa) /$ fin, and any extension of a nontrivial automorphism of $\mathcal{P}(\omega) /$ fin must also be nontrivial. In the case of $\mathcal{C}(\mathcal{H})$ this is not so clear, and in fact it is not yet known whether the existence of an outer automorphism of $\mathcal{C}(\mathcal{H})$, when $\mathcal{H}$ is nonseparable, is consistent with ZFC. However in the case where $\mathcal{H}$ is nonseparable there is more than one quotient of $\mathcal{B}(\mathcal{H})$ to consider. In this note we study some of these different quotients, and offer some alternatives results;

Theorem 1.1. Let $\mathcal{H}$ be a Hilbert space of some regular, uncountable dimension $\kappa$ and let $\mathcal{J}$ be the ideal in $\mathcal{B}(\mathcal{H})$ of operators whose range has dimension less than $\kappa$. If $2^{\kappa}=\kappa^{+}$, then the quotient $\mathcal{B}(\mathcal{H}) / \mathcal{J}$ has $2^{\kappa^{+}}$many outer automorphisms.

Theorem 1.2. Let $\mathcal{H}$ be a Hilbert space of dimension $\aleph_{1}$, let $\mathcal{J}$ be the ideal of operators on $\mathcal{H}$ whose range has dimension $<\aleph_{1}$, and let $\mathcal{K}$ be the ideal of compact operators. If $C H$ holds, then $\mathcal{J} / \mathcal{K}$ has $2^{\aleph_{1}}$-many outer automorphisms.

Theorem 1.1 is perhaps most striking in the case $\kappa=\aleph_{1}$, for in this case its only set-theoretic assumption, $2^{\aleph_{1}}=\aleph_{2}$, follows already from PFA. Hence in a model of PFA, there are many outer automorphisms of $\mathcal{B}(\mathcal{H}) / \mathcal{J}$, and yet no outer automorphisms of $\mathcal{B}(\mathcal{H}) / \mathcal{K}$.

Our notation is mostly standard. All Hilbert spaces considered are complex Hilbert spaces. When $\mathcal{H}$ is a Hilbert space, $\mathcal{B}(\mathcal{H})$ denotes the $\mathrm{C}^{*}$-algebra of bounded linear operators from $\mathcal{H}$ to $\mathcal{H}, \mathcal{K}(\mathcal{H})$ denotes the closed $*$-ideal in $\mathcal{B}(\mathcal{H})$ given by the compact operators on $\mathcal{H}$, and $\mathcal{J}(\mathcal{H})$ denotes the $*$-ideal of operators whose range has dimension strictly less than the dimension of $\mathcal{H}$. When the Hilbert space $\mathcal{H}$ is understood we will often drop it in our notation and just use $\mathcal{B}, \mathcal{K}$, and $\mathcal{J}$. Note that when $\mathcal{H}$ is nonseparable, $\mathcal{J}$ is already norm-closed, and

$$
\mathcal{K} \subset \mathcal{J} \subset \mathcal{B}
$$

If $x \in \mathcal{B}$ then we will use $[x]_{\mathcal{K}}$ and $[x]_{\mathcal{J}}$ to denote the quotients of $x$ by $\mathcal{K}(\mathcal{H})$ and $\mathcal{J}(\mathcal{H})$ respectively. When $A$ is a set, we will write $\ell^{2}(A)$ for the Hilbert space of square-summable functions $\xi: A \rightarrow \mathbb{C}$. We will also often write $\mathcal{B}(\mathcal{H})=\mathcal{B}_{A}, \mathcal{J}(\mathcal{H})=\mathcal{J}_{A}$, and $\mathcal{K}(\mathcal{H})=\mathcal{K}_{A}$ when $\mathcal{H}=\ell^{2}(A)$. When $A \subseteq B$ we will identify $\ell^{2}(A)$ with a closed subspace of $\ell^{2}(B)$ in the obvious way. Finally, if $A$ is a $\mathrm{C}^{*}$-algebra and $x$ is an element of $A$ then $\operatorname{Ad} x: A \rightarrow A$ is the map $a \mapsto x a x^{*}$. When $A$ has a multiplicative unit and $x$ is a unitary element of $A$, i.e. $x^{*} x=x x^{*}=1_{A}$, then $\operatorname{Ad} x$ is an automorphism of $A$. 


\section{LARGE IDEALS}

In this section we prove Theorem 1.1. Before beginning the proof we will need some notation;

Definition 2.1. If $C$ is club in $\kappa$, we define

$x \in \mathcal{D}[C] \Longleftrightarrow \forall \alpha \in C \quad \ell^{2}(\alpha)$ is an invariant subspace of $x$ and $x^{*}$

Note that $\mathcal{D}[C]$ is a $\mathrm{C}^{*}$-subalgebra of $\mathcal{B}_{\kappa}$, and in fact is a von Neumann subalgebra of $\mathcal{B}_{\kappa}$, though we will not use this latter fact. We also set down some convenient notation for the successor of an ordinal in a club;

Definition 2.2. If $C$ is club in $\kappa$ and $\alpha \in C$, then $\operatorname{succ}_{C}(\alpha)$ denotes the minimal element of $C$ strictly greater than $\alpha$.

Note that if $C$ is club in $\kappa$, then we have in fact

$x \in \mathcal{D}[C] \Longleftrightarrow \forall \alpha \in C \quad \ell^{2}\left(\left[\alpha, \operatorname{succ}_{C}(\alpha)\right)\right)$ is an invariant subspace of $x$

Finally, if $A, B \subseteq \kappa$ then we write $A \subseteq^{*} B$ if and only if $|A \backslash B|<\kappa$.

Lemma 2.3. For every $x \in \mathcal{B}_{\kappa}$, there is some club $C$ in $\kappa$ such that $x \in$ $\mathcal{D}[C]$.

Proof. Let $\theta$ be large and regular, and let $M_{\alpha}$, for $\alpha<\kappa$, be a club of elementary substructures of $H(\theta)$, each of size $<\kappa$, and with $x$ and $\ell^{2}(\kappa)$ in $M_{0}$. Then if $\delta=\sup \left(M_{\alpha} \cap \kappa\right)$, we clearly have that $\ell^{2}(\delta)$ is an invariant subspace of $x$, and such ordinals $\delta$ make up a club in $\kappa$.

Lemma 2.4. If $C \subseteq^{*} \widetilde{C}$ are clubs in $\kappa$, then $\mathcal{D}[\widetilde{C}] \subseteq \mathcal{J} \mathcal{D}[C]$, by which we mean

$$
\forall x \in \mathcal{D}[\widetilde{C}] \exists y \in \mathcal{D}[C] \quad x-y \in \mathcal{J}
$$

Proof. If $\gamma<\kappa$ is such that $C \cap[\gamma, \kappa) \subseteq \widetilde{C}$, then for every $\delta \in \widetilde{C}$,

$$
\delta \geq \gamma \Longrightarrow\left[\delta, \operatorname{succ}_{\widetilde{C}}(\delta)\right) \subseteq\left[\delta, \operatorname{succ}_{C}(\delta)\right)
$$

Thus if $x \in \mathcal{D}[\widetilde{C}]$, we see that $P x P \in \mathcal{D}[C]$, where $P$ is the projection onto the subspace $\ell^{2}([\gamma, \kappa))$.

Lemma 2.5. Let $C$ be club in $\kappa$ and let $u$ and $v$ be unitary operators on $\ell^{2}(\kappa)$, which are diagonal with respect to the standard basis; say $f, g: \kappa \rightarrow \mathbb{T}$ are the diagonal values of $u$ and $v$ respectively. Then $\operatorname{Ad}[u]_{\mathcal{J}}$ and $\operatorname{Ad}[v]_{\mathcal{J}}$ agree on $\mathcal{D}[C] / \mathcal{J}$ if and only if there is some $\epsilon<\kappa$ such that the map

$$
\xi \mapsto \frac{f(\xi)}{g(\xi)}=f(\xi) \overline{g(\xi)}
$$

is constant on each interval of the form $\left[\delta, \operatorname{succ}_{C}(\delta)\right)$ with $\delta \in C \cap[\epsilon, \kappa)$.

Proof. Let $h(\xi)=f(\xi) \overline{g(\xi)}$ for each $\xi<\kappa$. We will write $(*)$ for the condition

$\exists \epsilon \forall \delta \in C \quad \delta \geq \epsilon \Longrightarrow h$ is constant on the interval $\left[\delta, \operatorname{succ}_{C}(\delta)\right)$

as in the conclusion of the lemma. Now, note that $u$ and $v$ are trivially in the algebra $\mathcal{D}[C]$. The following are equivalent; 
(1) $\operatorname{Ad}[u]_{\mathcal{J}}$ and $\operatorname{Ad}[v]_{\mathcal{J}}$ agree on $\mathcal{D}[C] / \mathcal{J}$,

(2) for each $x \in \mathcal{D}[C], u x u^{*}-v x v^{*}$ is in $\mathcal{J}$,

(3) $\left[v^{*} u\right]_{\mathcal{J}}$ is in the center of the algebra $\mathcal{D}[C] / \mathcal{J}$.

We will show that condition (3) holds if and only if $(*)$ holds. First suppose $(*)$ does not hold; then there is an unbounded subset $A$ of $C$, and sequences $\sigma_{\delta}, \tau_{\delta}$ indexed by $\delta \in A$, such that for each $\delta \in A, \delta \leq \sigma_{\delta}<\tau_{\delta}<\operatorname{succ}_{C}(\delta)$ and $h\left(\sigma_{\delta}\right) \neq h\left(\tau_{\delta}\right)$. Let $x$ be the operator defined by

$$
x\left(e_{\alpha}\right)= \begin{cases}e_{\sigma_{\delta}} & \alpha=\tau_{\delta} \text { for some } \delta \in A \\ e_{\tau_{\delta}} & \alpha=\sigma_{\delta} \text { for some } \delta \in A \\ 0 & \text { otherwise }\end{cases}
$$

Then $x \in \mathcal{D}[C]$, and for each $\delta \in A$,

$$
\left(v^{*} u x\right) e_{\sigma_{\delta}}=h\left(\tau_{\delta}\right) e_{\tau_{\delta}} \quad\left(x v^{*} u\right) e_{\sigma_{\delta}}=h\left(\sigma_{\delta}\right) e_{\tau_{\delta}}
$$

It follows that $v^{*} u x-x v^{*} u$ is not in the ideal $\mathcal{J}$, so condition (3) does not hold. Now suppose $(*)$ does hold, and choose $\epsilon$ as in this condition. If $x \in \mathcal{D}[C]$, then for all $\alpha \geq \epsilon$, if $\alpha \in\left[\delta\right.$, $\left.\operatorname{succ}_{C}(\delta)\right)$ where $\delta \in C$ then we have

$$
\left(v^{*} u x\right) e_{\alpha}=h(\alpha) x e_{\alpha}=\left(x v^{*} u\right) e_{\alpha}
$$

and it follows that $P\left(v^{*} u\right) P$ is in the center of $\mathcal{D}[C]$, where $P$ is the projection onto $\ell^{2}([\epsilon, \kappa))$.

We are now ready to prove Theorem 1.1 .

Proof. Let $\left\langle E_{\alpha} \mid \alpha \in \lim \left(\kappa^{+}\right)\right\rangle$enumerate the clubs in $\kappa$. We will construct a sequence of clubs $C_{s}$ in $\kappa$, and functions $f_{s}: \kappa \rightarrow \mathbb{T}$, indexed by $s \in 2^{<\kappa^{+}}$, such that

(1) If $s \subset t$, then $C_{t} \subseteq^{*} C_{s}$.

(2) If $s \subset t$, then there is an $\epsilon<\kappa$ such that for every $\delta \in C_{s}$ with $\delta \geq \epsilon$, the function $f_{s} \overline{f_{t}}$ is constant on the interval $\left[\delta, \operatorname{succ}_{C_{s}}(\delta)\right)$.

(3) For all $s, C_{s^{\urcorner} 0}=C_{s^{\urcorner} 1}$, and for unboundedly many $\delta \in C_{s^{\urcorner} 0}=$ $C_{s^{\wedge} 1}=C$, the function $f_{s^{\wedge} 0} \overline{f_{s\urcorner 1}}$ is not constant on $\left[\delta, \operatorname{succ}_{C}(\delta)\right)$.

(4) If $s$ has length some limit ordinal $\alpha<\kappa^{+}$, then $C_{s} \subseteq^{*} E_{\alpha}$.

Claim 2.6. This suffices.

Proof. For each $s \in 2^{<\kappa^{+}}$, let $u_{s}$ be the diagonal unitary in $\mathcal{B}_{\kappa}$ with diagonal elements given by $f_{s}$. For each $\zeta \in 2^{\kappa^{+}}$, and $x \in \mathcal{D}\left[C_{\zeta\lceil\alpha}\right]$, define

$$
\Phi_{\zeta}([x])=\left[u_{\zeta\lceil\alpha} x u_{\zeta\lceil\alpha}^{*}\right]
$$

By (11), (2), and Lemma 2.5, $\Phi_{\zeta}$ is well-defined on the union of the algebras $\mathcal{D}\left[C_{\zeta\lceil\alpha}\right] / \mathcal{J}$, over $\alpha<\kappa^{+}$; and by (4), and Lemma 2.3. it follows that $\Phi_{\zeta}$ is defined on all of $\mathcal{B}_{\kappa} / \mathcal{J}$. Since on each $\mathcal{D}\left[C_{\zeta\lceil\alpha}\right], \Phi_{\zeta}$ agrees with $\operatorname{Ad}\left[u_{\zeta\lceil\alpha}\right]$, $\Phi_{\zeta}$ is also an injective homomorphism. Similar arguments show that $\Phi_{\zeta}^{-1}$ is defined on all of $\mathcal{B}_{\kappa} / \mathcal{J}$, and hence $\Phi_{\zeta}$ is an automorphism of this quotient algebra. Finally, if $\zeta$ and $\eta$ are distinct members of $2^{\kappa^{+}}$, then by (3) and Lemma 2.5 we see that $\Phi_{\zeta}$ and $\Phi_{\eta}$ are distinct automorphisms. 
We construct $C_{s}$ and $f_{s}$ by induction on the length of $s \in 2^{<\kappa^{+}}$. It is useful to note that all the functions $f_{s}$ constructed in the following actually have range contained in $\{-1,+1\}$; when proving (2) and (3), then, we will drop all mention of the conjugation. In the base case we simply set $C_{\langle\rangle}=\kappa$ and $f_{\langle\rangle}(\alpha)=1$ for all $\alpha<\kappa$. For the successor case, let $s \in 2^{<\kappa^{+}}$be given. Set $C_{s^{\urcorner} 0}=C_{s^{\urcorner} 1}=\lim \left(C_{s}\right), f_{s^{\frown} 0}=f_{s}$, and

$f_{s^{\wedge} 1}(\alpha)= \begin{cases}-f_{s}(\alpha) & \text { if there is } \delta \in \lim \left(C_{s}\right) \text { such that } \delta \leq \alpha<\operatorname{succ}_{C_{s}}(\delta) \\ +f_{s}(\alpha) & \text { otherwise }\end{cases}$

Obviously, the function $f_{s} f_{s^{\frown} 0}=f_{s}^{2}$ is constant on each interval of $C_{s}$ (in fact it is constant on all of $\kappa$ ). The same holds for the function $f_{s} f_{s^{\wedge} 1}$; if $\delta \in$ $\lim \left(C_{s}\right)$ then this function has a constant value of -1 on all of $\left[\delta, \operatorname{succ}_{C_{s}}(\delta)\right)$, whereas if $\delta \in C_{s} \backslash \lim \left(C_{s}\right)$ then it has a constant value of +1 on this interval. Hence condition (2) is satisfied in the inductive step. As for condition (3), we note that for every $\delta \in \lim \left(C_{s}\right)$, the function $f_{s^{\wedge} 0} f_{s^{\wedge} 1}$ is not constant on the interval $\left[\delta, \operatorname{succ}_{\lim \left(C_{s}\right)}(\delta)\right)$, since this function has a value of -1 at $\delta$ and a value of +1 at $\operatorname{succ}_{C_{s}}(\delta)<\operatorname{succ}_{\lim \left(C_{s}\right)}(\delta)$. It remains to consider the limit case. Let $s \in 2^{<\kappa^{+}}$be given, and let $\alpha$ be the length of $s$. For $\beta<\alpha$, write $f_{\beta}=f_{s \uparrow \beta}$ and $C_{\beta}=C_{s \uparrow \beta}$. By the inductive hypothesis, for every $\beta<\gamma<\alpha$ there is an $\epsilon<\kappa$ such that

$$
\forall \delta \in C_{\beta} \quad \delta \geq \epsilon \Longrightarrow f_{\beta} f_{\gamma} \text { is constant on the interval }\left[\delta, \operatorname{succ}_{C_{\beta}}(\delta)\right)
$$

Let $\epsilon_{\beta}^{\gamma}$ be the minimal $\epsilon \in C_{\beta}$ satisfying the above. We will define $f_{s}$ and $C_{s}$ in two different ways based on the cofinality of $\alpha$. First, suppose $\theta=\operatorname{cf} \alpha<\kappa$, and let $\alpha_{\eta}$, for $\eta<\theta$, be an increasing and continuous sequence of ordinals which is cofinal in $\alpha$. Define

$$
C_{s}=\left(\bigcap_{\eta<\theta} C_{\alpha_{\eta}}\right) \cap E_{\alpha}
$$

It remains to define $f_{s}$ and show that condition 2 holds. Choose a uniform ultrafilter $\widetilde{\mathcal{U}}$ over $\theta$, and let $\mathcal{U}_{\alpha}$ be the ultrafilter over $\alpha$ defined in the usual way from $\widetilde{\mathcal{U}}$ using the sequence $\left\langle\alpha_{\eta} \mid \eta<\theta\right\rangle$. Now for each $\xi<\kappa$ define

$$
f_{s}(\xi)=\lim _{\beta \in \mathcal{U}_{\alpha}} f_{\beta}(\xi)
$$

Claim 2.7. For every $\beta<\alpha, f_{\beta} f_{s}$ is constant on each interval of a tail of intervals from $C_{\beta}$.

Proof. Fix $\beta<\alpha$, and let $\epsilon=\sup _{\eta<\theta} \epsilon_{\beta}^{\alpha_{\eta}} \in C_{\beta}$. Let $\delta \in C_{\beta}$ be given, and suppose $\delta \geq \epsilon$, but that $f_{\beta} f_{s}$ is not constant on $\left[\delta, \operatorname{succ}_{C_{\beta}}(\delta)\right)$; fix witnesses $\sigma<\tau$ in this interval, and say without loss of generality that $f_{\beta}(\sigma) f_{s}(\sigma)=+1$ but $f_{\beta}(\tau) f_{s}(\tau)=-1$. By the definition of $f_{s}$, there are 
$A_{0}, A_{1} \in \mathcal{U}_{\alpha}$ such that

$$
\begin{array}{ll}
\forall \gamma \in A_{0} & f_{\beta}(\sigma) f_{\gamma}(\sigma)=+1 \\
\forall \gamma \in A_{1} & f_{\beta}(\tau) f_{\gamma}(\tau)=-1
\end{array}
$$

Then if $\gamma \in A_{0} \cap A_{1}$ is larger than $\beta$ we have $f_{\beta}(\sigma) f_{\gamma}(\sigma)=+1$ and $f_{\beta}(\tau) f_{\gamma}(\tau)=-1$. By definition of $\mathcal{U}_{\alpha}$ we may choose such a $\gamma$ with $\gamma=\alpha_{\eta}$ for some $\eta<\theta$. But this contradicts the choice of $\epsilon_{\beta}^{\gamma}$, since $\delta \geq \epsilon>$ $\epsilon_{\beta}^{\alpha_{\eta}}$

Now consider the case where $\operatorname{cf} \alpha=\kappa$. Let $\alpha_{\eta}, \eta<\kappa$, be a continuous, increasing sequence of ordinals which is cofinal in $\alpha$. Put

$$
C_{s}=\left(\underset{\eta<\kappa}{\Delta} C_{\alpha_{\eta}}\right) \cap E_{\alpha}
$$

Again, it remains only to define $f_{s}$ and show that condition (2) holds. For this we define, for $\xi<\eta$,

$$
\rho_{\xi}^{\eta}=\min \left(C_{\alpha_{\xi}} \backslash\left(\xi \cup \epsilon_{\alpha_{\xi}}^{\alpha_{\eta}}\right)\right)
$$

and

$$
\epsilon(\eta)=\sup _{\xi<\eta} \rho_{\xi}^{\eta}
$$

Note that $\epsilon(\eta)$ is in $C_{\alpha_{\xi}}$ for each $\xi<\eta$. Define $f_{s}(\zeta)=f_{\alpha_{\eta}}(\zeta)$ whenever $\epsilon(\eta) \leq \zeta<\epsilon(\eta+1)$ for some $\eta<\kappa$, that is,

$$
f_{s}=\bigcup_{\eta<\kappa} f_{\alpha_{\eta}} \uparrow[\epsilon(\eta), \epsilon(\eta+1))
$$

Claim 2.8. For every $\beta<\alpha, f_{\beta} f_{s}$ is constant on a tail of intervals from $C_{\beta}$.

Proof. We will first prove that $f_{\alpha_{\xi}} f_{s}$ is constant on a tail of intervals from $C_{\alpha_{\xi}}$, for each $\xi<\kappa$. Let $\epsilon=\epsilon(\xi+1)$; then if $\delta \in C_{\alpha_{\xi}}$ and $\delta \geq \epsilon$, we have $\epsilon(\eta) \leq \delta<\operatorname{succ}_{\alpha_{\alpha_{\xi}}}(\delta) \leq \epsilon(\eta+1)$ for some $\eta>\xi$. Hence $f_{s}$ is equal to $f_{\alpha_{\eta}}$ on the interval $\left[\delta, \operatorname{succ}_{\alpha_{\alpha_{\xi}}}(\delta)\right)$. Since $\delta \geq \epsilon(\eta) \geq \epsilon_{\alpha_{\xi}}^{\alpha_{\eta}}$, we see that $f_{\alpha_{\xi}} f_{s}$ is constant on this interval, as required.

Now let $\beta<\alpha$ be given, and choose $\xi<\kappa$ such that $\beta<\alpha_{\xi}$. By the above, there is an $\epsilon_{0}$ such that $f_{\alpha_{\xi}} f_{s}$ is constant on each interval of $C_{\alpha_{\xi}}$ beyond $\epsilon_{0}$. Let $\epsilon_{1}=\epsilon_{\beta}^{\alpha_{\xi}}$, and choose an $\epsilon_{2}$ such that $C_{\alpha_{\xi}} \cap\left[\epsilon_{2}, \kappa\right) \subseteq C_{\beta}$. It follows that with $\epsilon=\max \left\{\epsilon_{0}, \epsilon_{1}, \epsilon_{2}\right\}$ we have

$$
\forall \delta \in C_{\beta} \quad \delta \geq \epsilon \Longrightarrow f_{\beta} f_{s} \text { is constant on the interval }\left[\delta, \operatorname{succ}_{C_{\beta}}(\delta)\right)
$$

Thus we have proven condition (2) in this case, and this finishes the proof of the theorem. 


\section{SMALL IDEALS}

In this section we work with the Hilbert space $\mathcal{H}=\ell^{2}\left(\omega_{1}\right)$. Hence the ideals of $\mathcal{B}(\mathcal{H})$ are exactly

$$
0 \subset \mathcal{K} \subset \mathcal{J} \subset \mathcal{B}
$$

Letting $\mathcal{C}(\mathcal{L})$ denote the usual Calkin algebra over $\mathcal{L}$, i.e. $\mathcal{B}(\mathcal{L}) / \mathcal{K}(\mathcal{L})$, it follows that

$$
\mathcal{J} / \mathcal{K}=\bigcup_{\alpha<\omega_{1}} \mathcal{C}\left(\ell^{2}(\alpha)\right) \subset \mathcal{C}\left(\ell^{2}\left(\omega_{1}\right)\right)
$$

We will shortly prove Theorem 1.2, in a slightly stronger form; namely, assuming $\mathrm{CH}$, there is an automorphism $\Psi$ of the quotient $\mathcal{J} / \mathcal{K}$ whose restriction to each subalgebra $\mathcal{C}\left(\ell^{2}(\alpha)\right)$ is an outer automorphism. It follows also that $\Psi$ cannot be the restriction of an inner automorphism of $\mathcal{B} / \mathcal{K}$. Before we start, we will need a special case of Lemma 4.1 from [2]. We include its proof here for completeness.

Lemma 3.1. Let $\Phi$ be an automorphism of $\mathcal{C}(\mathcal{H})$, where $\mathcal{H}$ is any Hilbert space. Then $\Phi$ is inner if and only if it is inner on some subspace $\mathcal{L}$ of $\mathcal{H}$ of the same dimension.

Proof. Let $\mathcal{L}$ be a subspace of $\mathcal{H}$ of the same dimension. Then there is an isometry $U: \mathcal{H} \rightarrow \mathcal{L}$; let $u$ be its image in $\mathcal{C}(\mathcal{H})$. Suppose $\Phi$ is implemented by conjugation by $v$ on $\mathcal{C}(\mathcal{L})$; then for any $x \in \mathcal{C}(\mathcal{H})$,

$$
\Phi(x)=\Phi\left(u^{*} u x u^{*} u\right)=\Phi(u)^{*} v \Phi(u) x \Phi(u)^{*} v^{*} \Phi(u)
$$

and hence $\Phi$ is implemented by conjugation by $\Phi(u)^{*} v \Phi(u)$ on all of $\mathcal{C}(\mathcal{H})$.

Theorem 3.2. Assume $C H$. Then there are $2^{\aleph_{1}}$-many outer automorphisms of $\mathcal{J} / \mathcal{K}$. Moreover, each of these automorphisms is outer in a strong sense, namely each is outer when restricted to any $\mathcal{C}\left(\ell^{2}(\alpha)\right), \alpha<\omega_{1}$.

Proof. Let $\Phi$ be an automorphism of $\mathcal{C}\left(\ell^{2}(\omega)\right)$. Let $f_{\alpha}: \alpha \rightarrow \omega, \alpha<\omega_{1}$, be a sequence of injections satisfying

$$
\forall \alpha<\beta<\omega_{1} \quad f_{\beta}\left\lceil\alpha={ }^{*} f_{\alpha}\right.
$$

for every $\alpha<\beta<\omega_{1}$. Set $A_{\alpha}=\operatorname{ran}\left(f_{\alpha}\right)$, let $U_{\alpha}: \ell^{2}(\alpha) \rightarrow \ell^{2}\left(A_{\alpha}\right)$ be the unitary operator induced by $f_{\alpha}$, and let $u_{\alpha}$ be its image in $\mathcal{C}\left(\ell^{2}\left(\omega_{1}\right)\right)$. Let $\Psi$ be the unique automorphism of $\mathcal{J} / \mathcal{K}$ such that

$$
\forall \alpha<\omega_{1} \quad\left(\operatorname{Ad} u_{\alpha}\right) \circ \Psi=\Phi \circ\left(\operatorname{Ad} u_{\alpha}^{*}\right)
$$

Condition (11) ensures that such a $\Psi$ exists, and verifying that $\Psi$ is an automorphism is trivial. Lemma 3.1 implies that if $\Phi$ is outer, then $\Phi$ is also outer on every $\ell^{2}\left(A_{\alpha}\right)$, and hence $\Psi$ is outer on every $\ell^{2}(\alpha)$. By the main theorem of [4], there are $2^{\aleph_{1}}$-many outer automorphisms of $\mathcal{C}\left(\ell^{2}(\omega)\right)$, and hence $2^{\aleph_{1}}$-many outer automorphisms of $\mathcal{J} / \mathcal{K}$. 


\section{REFERENCES}

[1] Ilijas Farah, All automorphisms of all Calkin algebras, Math. Res. Lett. 18 (2011), no. 3, 489-503. MR 2802582 (2012e:03104)

[2] - All automorphisms of the Calkin algebra are inner, Ann. of Math. (2) 173 (2011), no. 2, 619-661. MR 2776359 (2012e:03103)

[3] Justin Tatch Moore, The proper forcing axiom, Proceedings of the International Congress of Mathematicians. Volume II (New Delhi), Hindustan Book Agency, 2010, pp. 329. MR 2827783 (2012g:03139)

[4] N. Christopher Phillips and Nik Weaver, The Calkin algebra has outer automorphisms, Duke Math. J. 139 (2007), no. 1, 185-202. MR 2322680 (2009a:46123)

[5] Walter Rudin, Homogeneity problems in the theory of Čech compactifications, Duke Math. J. 23 (1956), 409-419. MR 0080902 (18,324d)

[6] Saharon Shelah, Proper forcing, Lecture Notes in Mathematics, vol. 940, SpringerVerlag, Berlin, 1982. MR 675955 (84h:03002)

[7] Saharon Shelah and Juris Steprāns, PFA implies all automorphisms are trivial, Proc. Amer. Math. Soc. 104 (1988), no. 4, 1220-1225. MR 935111 (89e:03080)

Ilijas Farah: Department of Mathematics and Statistics, York University, 4700 Keele Street, North York, Ontario, Canada, M3J 1P3; and Matematicki Institut, Kneza Mihaila 34, Belgrade, Serbia.

E-mail address: ifarah@mathstat.yorku.ca

Paul McKenney: Department of Mathematical Sciences, Carnegie Mellon University, Pittsburgh, USA

E-mail address: pmckenne@andrew.cmu.edu

Ernest Schimmerling: Department of Mathematical Sciences, Carnegie MelLON University, PitTsburgh, USA

E-mail address: eschimme@andrew.cmu.edu 